\title{
Study of Thermodynamics in Generalized Holographic and Ricci Dark Energy Models
}

\author{
Samarpita Bhattacharya* and Ujjal Debnath ${ }^{\dagger}$ \\ Department of Mathematics, Bengal Engineering and Science University, Shibpur, Howrah-711 103, India.
}

(Dated: November 12, 2018)

\begin{abstract}
We have considered the flat FRW model of the universe which is filled with the combination of dark matter and dark energy. Here we have considered two types of dark energy models: (i) Generalized holographic and (ii) generalized Ricci dark energies. The general descriptions of first law and generalized second law (GSL) of thermodynamics have studied on the apparent horizon, particle horizon and event horizon of the universe. We have shown that the first law and GSL are always valid on apparent horizon and first law can not be satisfied on the particle and event horizons in Einstein's gravity. These results are always true for any types of dark energy models i.e., these results do not depend on the dark energy models in Einstein's gravity. But the GSL completely depends on the choices of dark energy models in Einstein's gravity. Here we have discussed the validity of GSL in Generalized holographic and generalized Ricci dark energy models. On the particle horizon GSL may be satisfied but on the event horizon the GSL can not be satisfied for both the dark energy models. Also we have considered the Generalized holographic dark energy and generalized Ricci dark energy as the original holographic dark energy, so in this situation we have calculated the expression of the radius of the horizon $L$. On this horizon, we have shown that the first law can not be satisfied. Finally, on the horizon of radius $L$, we have found that the GSL can not be satisfied for both the dark energy models.
\end{abstract}

\section{INTRODUCTION}

Recent astrophysical data from distant Ia supernovae observations [1, 2] show that the current Universe is not only expanding, but also it is accelerating due to some kind of negative-pressure form of matter known as dark energy [3,4]. This mysterious fluid is believed to dominate over the matter content of the Universe by $70 \%$ and to have enough negative pressure as to drive present day acceleration. However, the nature of dark energy is still unknown, and people have proposed some candidates to describe it. There are different candidates to play the role of the dark energy. The most traditional candidate is a non-vanishing cosmological constant which can also be though of as a perfect fluid satisfying the equation of state $p=-\rho$. The next simple model proposed for dark energy is the quintessence [5] which is a dynamical scalar field which slowly rolls down in a at enough potential. There are different alternative theories for the dynamical dark energy scenario which have been proposed by people to interpret the accelerating universe like K-essence [6], tachyonic field [7], Chaplygin gas [8], phantom field [9], etc. Next we will discuss another two types of dark energy candidates i.e., holographic and Ricci dark energies.

In quantum field theory a short distance (UV) cut-off is related to a long distance (IR) cut-off $L$ due to the limit set by formation of a black hole, which results in an upper bound on the zero-point energy density [10]. It is to be noted that the size $L$ should not exceed the mass of a black hole with the same size [11], i.e. $L^{3} \Lambda^{4} \leq L M_{p}^{2}$ where $\Lambda$ is the ultraviolet (UV) cut-off of the effective quantum field theory. The largest IR cutoff $L$ is chosen by saturating the inequality, so that the holographic dark energy density [12] may be defined by $\rho_{\Lambda}=3 c^{2} M_{p}^{2} L^{-2}$ where $M_{p}=1 / \sqrt{8 \pi G}$ is the reduced Planck mass and $c$ is a numerical constant characterizing all of the uncertainties of the theory, whose value can only be determined by observations. On the basis of the holographic principle proposed by [13] several others have studied holographic model for dark energy [14]. Employment of Friedman equation $\rho=3 M_{p}^{2} H^{2}$ where $\rho$ is the total energy density and taking $L=H^{-1}$ one can find $\rho_{m}=3\left(1-c^{2}\right) M_{p}^{2} H^{2}$. Thus either $\rho_{m}$ or $\rho_{\Lambda}$ behaves like $H^{2}$. If we take $L$ as the size of the current universe, say, the Hubble radius $\frac{1}{H}$ then the dark energy density will be close to the observational result. There are other type of dark energy i.e., Ricci dark energy, which is a kind of holographic dark energy [15] taking the square root of the inverse Ricci scalar as its infrared cutoff and this model is also phenomenologically viable.

\footnotetext{
* samarpita_sarbajna@yahoo.co.in

† ujjaldebnath@yahoo.com , ujjal@iucaa.ernet.in
} 
Gao et al [16] proposed the dark energy density proportional to the Ricci scalar $R$ i.e., $\rho_{X} \propto R$. This model works fairly well in fitting the observational data, and it could also help to understand the coincidence problem. There are several works on this Ricci dark energy model [17].

Here we shall consider two types of dark energy models: (i) generalized holographic and (ii) generalized Ricci dark energy models [18]. In Section II, we have found some solutions if the universe is filled with dark matter and generalized holographic/Ricci dark energy. General prescriptions of first law and GSL of thermodynamics have been presented in section III. We have shown that the first law and GSL are always valid on apparent horizon and first law can not be satisfied on the particle and event horizons in Einstein's gravity. These results are always true for any types of dark energy models i.e., these results do not depend on the dark energy models in Einstein's gravity. But the GSL completely depends on the choices of dark energy models in Einstein's gravity. There are several works on the validity of GSL on the particle and event horizons in various dark energy models [19, 20, 21]. Here we shall discuss the validity of GSL in Generalized holographic and generalized Ricci dark energy models in section IV. Also if we consider the generalized holographic dark energy and generalized Ricci dark energy as the original holographic dark energy, then our aim is to check the validity of laws of thermodynamics on the horizon of radius $L$. Some conclusions have been drawn in last section.

\section{GENERALIZED HOLOGRAPHIC AND RICCI DARK ENERGY MODELS}

The Einstein's field equations for homogeneous, isotropic and flat FRW universe are given by

$$
H^{2}=\frac{8 \pi}{3} \rho
$$

and

$$
\dot{H}=-4 \pi(\rho+p)
$$

where $H\left(=\frac{\dot{a}}{a}\right)$ is the Hubble parameter (choosing $G=c=1$ ) and $\rho$ and $p$ be the energy density and pressure of the fluid respectively. The conservation equation is given by

$$
\dot{\rho}+3 H(\rho+p)=0
$$

Now let us consider the fluid is a combination of dark matter and dark energy i.e., $\rho=\rho_{m}+\rho_{D E}$ and $p=p_{m}+p_{D E}$ with $p_{m}=0$. Assuming there is no interaction between dark matter and dark energy, so they are separately conserved. Thus the the conservation equation (3) becomes

$$
\dot{\rho}_{m}+3 H \rho_{m}=0
$$

and

$$
\dot{\rho}_{D E}+3 H\left(\rho_{D E}+p_{D E}\right)=0
$$

From equation (4), we get the energy density of matter as

$$
\rho_{m}=\rho_{m_{0}}(1+z)^{3}
$$

where $\rho_{m_{0}}$ is an integration constant which gives the present value of the dark energy density and $z=\frac{1}{a}-1$ is the redshift.

Recently, Xu et al [18] proposed two types of dark energy models, i.e., generalized holographic and generalized Ricci dark energy models as follows: 
(i) Generalized Holographic Dark Energy Model (GHDEM): The energy density of GHDEM is given by,

$$
\rho_{h}=\rho_{D E}=\frac{3 c^{2}}{8 \pi} H^{2} f\left(R / H^{2}\right)
$$

where $c$ is a numerical constant and $f(x)$ is a positive function defined as, $f(x)=\alpha x+(1-\alpha), \alpha$ is a constant. Here $R$ is the Ricci scalar and its expression for flat universe is given by

$$
R=-6\left(\dot{H}+2 H^{2}\right)
$$

When $\alpha=0$ then $f(x)=1$, we recover the energy densities of original holographic dark energy. Also when $\alpha=1$ then $f(x)=x$, we recover the energy density of original Ricci dark energy.

So the energy density $\rho_{h}$ becomes

$$
\rho_{h}=\frac{3 c^{2}}{8 \pi}\left[(1-13 \alpha) H^{2}-6 \alpha \dot{H}\right]
$$

Solving (1), (6) and (9) we get

$$
H^{2}=\frac{8 \pi \rho_{m_{0}}(1+z)^{3}}{3\left(1-c^{2}+4 \alpha c^{2}\right)}+H_{0}^{2}(1+z)^{\frac{c^{2}(13 \alpha-1)+1}{3 \alpha c^{2}}}
$$

where, $H_{0}$ is an integration constant. Differentiating (10) w.r.t. $t$, we get

$$
\dot{H}=-\frac{8 \pi \rho_{m_{0}}(1+z)^{3}}{2\left(1-c^{2}+4 \alpha c^{2}\right)}+\frac{c^{2}(1-13 \alpha)-1}{6 \alpha c^{2}} H_{0}^{2}(1+z)^{\frac{c^{2}(13 \alpha-1)+1}{3 \alpha c^{2}}}
$$

From equation (8), we obtain the Ricci scalar $R$ as,

$$
R=-\frac{8 \pi \rho_{m_{0}}(1+z)^{3}}{\left(1-c^{2}+4 \alpha c^{2}\right)}-\frac{c^{2}(1-7 \alpha)-1}{3 \alpha c^{2}} H_{0}^{2}(1+z)^{\frac{c^{2}(13 \alpha-1)+1}{3 \alpha c^{2}}}
$$

So from equation (1) and (2), we can found the expressions for density $\rho_{h}$ and pressure $p_{h}$ as,

$$
\rho_{h}=\frac{c^{2}(1-4 \alpha) \rho_{m_{0}}(1+z)^{3}}{\left(1-c^{2}+4 \alpha c^{2}\right)}+\frac{3}{8 \pi} H_{0}^{2}(1+z)^{\frac{c^{2}(13 \alpha-1)+1}{3 \alpha c^{2}}}
$$

and

$$
p_{h}=\frac{c^{2}(4 \alpha-1)+1}{24 \pi \alpha c^{2}} H_{0}^{2}(1+z)^{\frac{c^{2}(13 \alpha-1)+1}{3 \alpha c^{2}}}
$$

(ii) Generalized Ricci Dark Energy Model (GRDEM): The energy density of GRDEM is given by,

$$
\rho_{r}=\frac{3 c^{2}}{8 \pi} R g\left(H^{2} / R\right)
$$

where $g(y)$ is a positive function defined as, $g(y)=\beta y+(1-\beta), \beta$ is a constant. When $\beta=0$ then $g(y)=1$, we recover the energy density of original Ricci dark energy. Also when $\beta=1$ then $g(y)=x$, we recover the energy density of original holographic dark energy.

Now comparing (7) and (15), we see that when $\beta=1-\alpha$ the generalized Ricci dark energy reduces to the generalized holographic dark energy and vice versa. If we replace $\alpha$ by $(1-\beta)$ in equations (9) - (14), we will get similar solutions for generalized Ricci dark energy model. Therefore, equations (9) - (14) are also the solutions of generalized Ricci dark energy model with $\alpha=1-\beta$. 


\section{FIRST LAW AND GENERALIZED SECOND LAW OF THERMODYNAMICS (GSL): GENERAL PRESCRIPTION}

We consider the FRW universe as a thermodynamical system with the horizon surface as a boundary of the system. Here, we study the validity of first law and generalized second law (GSL) of thermodynamics on apparent, particle and event horizons. To study the generalized second law (GSL) of thermodynamics through the universe we deduce the expression for normal entropy using the Gibb's law of thermodynamics [22]

$$
T_{X} d S_{I}=p d V+d\left(E_{X}\right)
$$

where, $S_{I}, p, V$ and $E_{X}$ are respectively entropy, pressure, volume and internal energy within the apparent/particle/event horizon and $T_{X}$ is the temperature on the apparent horizon $(X=A) /$ particle horizon $(X=P)$ /event horizon $(X=E)$. Here the expression for internal energy can be written as $E_{X}=\rho V$. Now the volume of the sphere is $V=\frac{4}{3} \pi R_{X}^{3}$, where $R_{X}$ is the radius of the apparent horizon $\left(R_{A}\right) /$ particle horizon $\left(R_{P}\right)$ /event horizon $\left(R_{E}\right)$ defined by [22] (see also our previous work [24])

$$
\begin{gathered}
R_{A}=\frac{1}{H} \\
R_{P}=a \int_{0}^{t} \frac{d t}{a}=\frac{1}{1+z} \int_{z}^{\infty} \frac{d z}{H}
\end{gathered}
$$

and

$$
R_{E}=a \int_{t}^{\infty} \frac{d t}{a}=\frac{1}{1+z} \int_{-1}^{z} \frac{d z}{H}
$$

which immediately give (after differentiation)

$$
\dot{R}_{P}=H R_{P}+1
$$

and

$$
\dot{R}_{E}=H R_{E}-1
$$

The temperature and the entropy on the apparent/particle/event horizon are also given by

$$
T_{X}=\frac{1}{2 \pi R_{X}}
$$

and

$$
S_{X}=\pi R_{X}^{2}
$$

Next, we shall examine the validity of first law and GSL on the apparent horizon, particle horizon and event horizon.

- First law on the apparent horizon, particle horizon and event horizon:

The amount of the energy crossing on the apparent/particle/event horizon is [23] given by

$$
-d E_{X}=4 \pi R_{X}^{3} H T_{\mu \nu} k^{\mu} k^{\nu} d t=4 \pi R_{X}^{3} H(\rho+p) d t=-H \dot{H} R_{X}^{3} d t
$$

The first law of thermodynamics on the apparent/particle/event horizon is defined as follows: 


$$
-d E_{X}=T_{X} d S_{X}
$$

On the apparent horizon, we have (using (17), (22), (23) and (24))

$$
-d E_{A}=-H \dot{H} R_{A}^{3} d t=-\frac{\dot{H}}{H^{2}} d t
$$

and

$$
T_{A} d S_{A}=-\dot{R}_{A} d t=-\frac{\dot{H}}{H^{2}} d t
$$

The equations (26) and (27) imply,

$$
-d E_{A}=T_{A} d S_{A}
$$

This shows that the first law is always satisfied on the apparent horizon in Einstein's gravity.

On the particle horizon, we have (using (18), (20), (22), (23) and (24))

$$
-d E_{P}=-H \dot{H} R_{P}^{3} d t
$$

and

$$
T_{P} d S_{P}=\dot{R}_{P} d t=\left(H R_{P}+1\right) d t
$$

From equations (29) and (30), we get

$$
-d E_{P}=T_{P} d S_{P}-\left(H \dot{H} R_{P}^{3}+H R_{P}+1\right) d t
$$

The second term on the r.h.s. of (31) is time dependent, so this term cannot become zero during certain stage of the evolution of the universe. Thus we may conclude that

$$
-d E_{P} \neq T_{P} d S_{P}
$$

So on the particle horizon, first law of thermodynamics can not be satisfied in Einstein's gravity.

On the event horizon, we have (using (19), (21), (22), (23) and (24))

$$
-d E_{E}=-H \dot{H} R_{E}^{3} d t
$$

and

$$
T_{E} d S_{E}=\dot{R}_{E} d t=\left(H R_{E}-1\right) d t
$$

From equations (33) and (34), we get

$$
-d E_{E}=T_{E} d S_{E}-\left(H \dot{H} R_{E}^{3}+H R_{E}-1\right) d t
$$

The second term on the r.h.s. is time dependent, so this term cannot become zero during certain stage of the evolution of the universe. Thus we may conclude that 


$$
-d E_{E} \neq T_{E} d S_{E}
$$

So on the event horizon, first law of thermodynamics can not be satisfied in Einstein's gravity.

- GSL on the apparent horizon, particle horizon and event horizon:

The rate of change of internal entropy and total entropy are obtained as (from (16), (22) and (23))

$$
\dot{S}_{I}=\frac{\dot{H} R_{X}^{2}\left(H R_{X}-\dot{R}_{X}\right)}{T_{X}}
$$

and

$$
\dot{S}_{I}+\dot{S}_{X}=2 \pi R_{X}\left[\dot{H} R_{X}^{2}\left(H R_{X}-\dot{R}_{X}\right)+\dot{R}_{X}\right]
$$

The GSL states that total entropy can not be decreased i.e.,

$$
\dot{S}_{I}+\dot{S}_{X} \geq 0 \text { i.e., } \dot{H} R_{X}^{2}\left(H R_{X}-\dot{R}_{X}\right)+\dot{R}_{X} \geq 0
$$

On the apparent horizon, the rate of change of total entropy is (using (17) and (38))

$$
\dot{S}_{I}+\dot{S}_{A}=2 \pi R_{A}\left[\dot{H} R_{A}^{2}\left(H R_{A}-\dot{R}_{A}\right)+\dot{R}_{A}\right]=\frac{2 \pi \dot{H}^{2}}{H^{5}} \geq 0
$$

So GSL always satisfied on the apparent horizon in Einstein's gravity.

On the particle horizon, the rate of change of total entropy is (using (20) and (38))

$$
\dot{S}_{I}+\dot{S}_{P}=2 \pi R_{P}\left[\dot{H} R_{P}^{2}\left(H R_{P}-\dot{R}_{P}\right)+\dot{R}_{P}\right]=2 \pi R_{P}\left(-\dot{H} R_{P}^{2}+H R_{P}+1\right)
$$

So GSL may be satisfied on the particle horizon if the following condition holds:

$$
-\dot{H} R_{P}^{2}+H R_{P}+1 \geq 0
$$

For quintessence model, $\dot{H}<0$, so GSL always satisfied. But for phantom model, $\dot{H}>0$, GSL may or may not be satisfied. For phantom crossing model, the GSL satisfied in the initial stage but in the late stage the GSL may not be satisfied during evolution of the universe on the particle horizon.

On the event horizon, the rate of change of total entropy is (using (21) and (38))

$$
\dot{S}_{I}+\dot{S}_{E}=2 \pi R_{E}\left[\dot{H} R_{E}^{2}\left(H R_{E}-\dot{R}_{E}\right)+\dot{R}_{E}\right]=2 \pi R_{E}\left(\dot{H} R_{E}^{2}+H R_{E}-1\right)
$$

So GSL may be satisfied on the event horizon if the following condition holds:

$$
\dot{H} R_{E}^{2}+H R_{E}-1 \geq 0
$$

For quintessence model $(\dot{H}<0)$ or phantom model $\dot{H}>0$ or phantom crossing model, we can not draw any definite conclusion for validity of GSL on event horizon.

So from above discussions, we may conclude that the validity of first law is independent of dark energy model in Einstein's gravity on apparent horizon, particle horizon and event horizon and also validity of GSL is independent of dark energy model in Einstein's gravity on apparent horizon. But validity of GSL is completely depends on the dark energy models in Einstein's gravity on particle and event horizons. Hence in the next section we shall discuss the validity of GSL on particle and event horizons for generalized holographic and generalized Ricci dark energy models. 


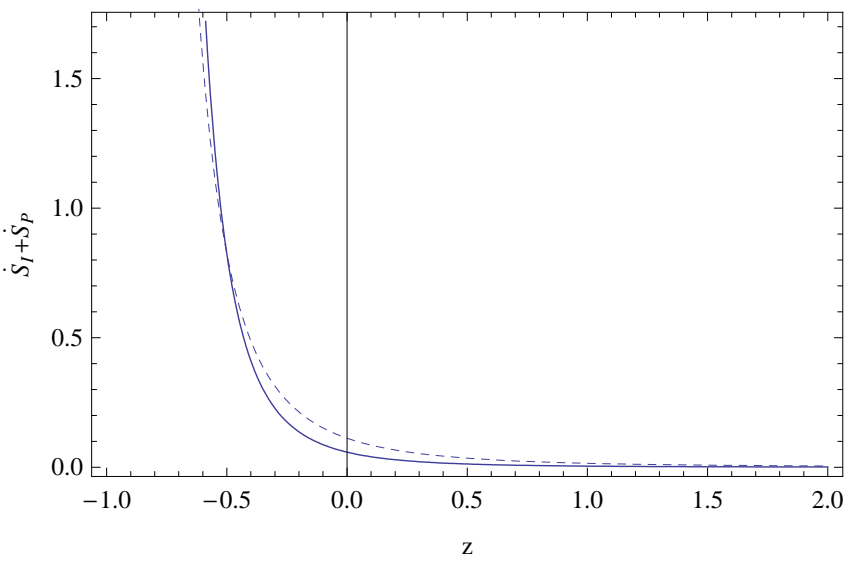

Fig.1

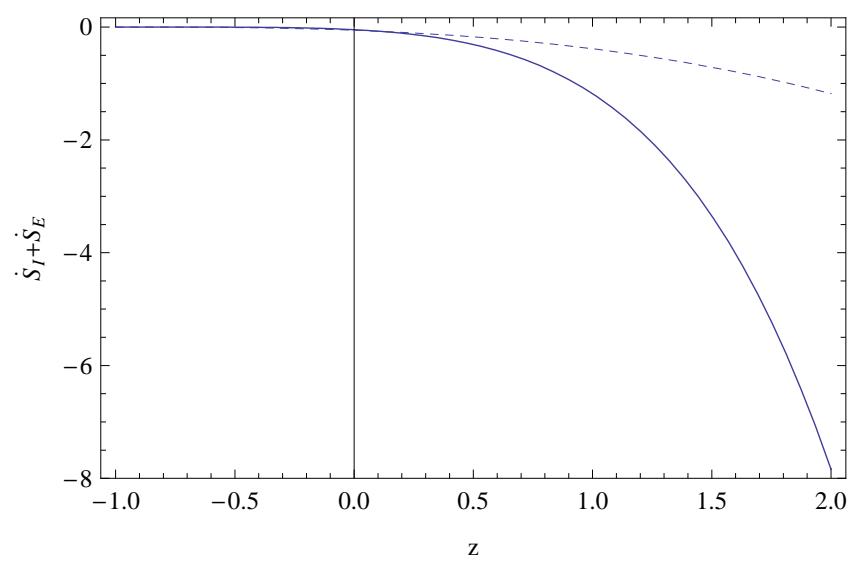

Fig.2

Figs. 1 and 2 show the variations of $\left(\dot{S}_{I}+\dot{S}_{P}\right)$ and $\left(\dot{S}_{I}+\dot{S}_{E}\right)$ respectively against redshift $z$ for $c=.5, \rho_{m_{0}}=1, \alpha=.7, \beta=.7, H_{0}=70$. The dash line and non-dash line represent for the generalized holographic and generalized Ricci dark energy models respectively.

\section{VALIDITY OF GSL ON PARTICLE AND EVENT HORIZONS IN GENERALIZED HOLOGRAPHIC AND RICCI DARK ENERGY MODELS}

In generalized holographic/Ricci dark energy model, the radii of the particle and event horizons in terms of redshift $z$ can be written as (using (10), (18) and (19))

$$
R_{P}=\frac{1}{1+z} \int_{z}^{\infty} \frac{d z}{H}=\frac{1}{1+z} \int_{z}^{\infty}\left[\frac{8 \pi \rho_{m_{0}}(1+z)^{3}}{3\left(1-c^{2}+4 \alpha c^{2}\right)}+H_{0}^{2}(1+z)^{\frac{c^{2}(13 \alpha-1)+1}{3 \alpha c^{2}}}\right]^{-\frac{1}{2}} d z
$$

and

$$
R_{E}=\frac{1}{1+z} \int_{-1}^{z} \frac{d z}{H}=\frac{1}{1+z} \int_{-1}^{z}\left[\frac{8 \pi \rho_{m_{0}}(1+z)^{3}}{3\left(1-c^{2}+4 \alpha c^{2}\right)}+H_{0}^{2}(1+z)^{\frac{c^{2}(13 \alpha-1)+1}{3 \alpha c^{2}}}\right]^{-\frac{1}{2}} d z
$$

On the particle horizon, the rate of change of total entropy is (using (10) and (41)) obtained as

$$
\begin{aligned}
& \dot{S}_{I}+\dot{S}_{P}=2 \pi R_{P}\left(-\dot{H} R_{P}^{2}+H R_{P}+1\right) \\
&=2 \pi R_{P}\left[\left\{\frac{8 \pi \rho_{m_{0}}(1+z)^{3}}{2\left(1-c^{2}+4 \alpha c^{2}\right)}-\frac{c^{2}(1-13 \alpha)-1}{6 \alpha c^{2}} H_{0}^{2}(1+z)^{\frac{c^{2}(13 \alpha-1)+1}{3 \alpha c^{2}}}\right\} R_{P}^{2}\right. \\
&\left.+\left\{\frac{8 \pi \rho_{m_{0}}(1+z)^{3}}{3\left(1-c^{2}+4 \alpha c^{2}\right)}+H_{0}^{2}(1+z)^{\frac{c^{2}(13 \alpha-1)+1}{3 \alpha c^{2}}}\right\}^{\frac{1}{2}} R_{P}+1\right]
\end{aligned}
$$

Also, on the event horizon, the rate of change of total entropy is (using (10) and (43)) obtained as 


$$
\begin{aligned}
& \dot{S}_{I}+\dot{S}_{E}=2 \pi R_{E}\left(\dot{H} R_{E}^{2}+H R_{E}-1\right) \\
&=2 \pi R_{E}\left[\left\{-\frac{8 \pi \rho_{m_{0}}(1+z)^{3}}{2\left(1-c^{2}+4 \alpha c^{2}\right)}+\frac{c^{2}(1-13 \alpha)-1}{6 \alpha c^{2}} H_{0}^{2}(1+z)^{\frac{c^{2}(13 \alpha-1)+1}{3 \alpha c^{2}}}\right\} R_{E}^{2}\right. \\
& \\
&\left.+\left\{\frac{8 \pi \rho_{m_{0}}(1+z)^{3}}{3\left(1-c^{2}+4 \alpha c^{2}\right)}+H_{0}^{2}(1+z)^{\frac{c^{2}(13 \alpha-1)+1}{3 \alpha c^{2}}}\right\}^{\frac{1}{2}} R_{E}-1\right]
\end{aligned}
$$

The rate of change of total entropies for particle and event horizons have drawn in figs. 1 and 2 respectively. So Figs. 1 and 2 show the variations of $\left(\dot{S}_{I}+\dot{S}_{P}\right)$ and $\left(\dot{S}_{I}+\dot{S}_{E}\right)$ respectively against redshift $z$ for $c=.5, \rho_{m_{0}}=1, \alpha=.7, \beta=.7, H_{0}=70$. The dash line and non-dash line represent for the generalized holographic and generalized Ricci dark energy models respectively. From the figures, we see that the GSL may be satisfied on the particle horizon for generalized holographic and Ricci dark energy models, but on the event horizon GSL can not be satisfied for these two dark energy models.

\section{GENERALIZED HOLOGRAPHIC/RICCI AS AN ORIGINAL HOLOGRAPHIC DARK ENERGY MODEL: THERMODYNAMICS}

We consider here that the generalized holographic/Ricci dark energy model as an original holographic dark energy model. For this purpose, we will compare the energy densities between generalized holographic/Ricci and original holographic dark energies. Now if we compare the energy density of holographic dark energy which is given in (7) with the original holographic model with energy density,

$$
\rho_{\Lambda}=\frac{3 c^{2}}{8 \pi} L^{-2}
$$

then we get,

$$
L^{2}=\frac{1}{\alpha R+(1-\alpha) H^{2}}
$$

which can be written in the simplified form (using (10) and (12)):

$$
L^{2}=\frac{3 c^{2}\left(1-c^{2}+4 \alpha c^{2}\right)}{8 c^{2}(1-4 \alpha) \pi \rho_{m_{0}}(1+z)^{3}+\left[2 c^{2}(1+2 \alpha)+1\right]\left(1-c^{2}+4 \alpha c^{2}\right) H_{0}^{2}(1+z)^{\frac{c^{2}(13 \alpha-1)+1}{3 \alpha c^{2}}}}
$$

Here, we will try to apply the usual definition of the temperature and entropy on the horizon of radius $L$ and examine the validity of first and the second laws of thermodynamics. The temperature and the entropy on the horizon are (similar to (22) and (23)) [25]

$$
T_{L}=\frac{1}{2 \pi L}
$$

and

$$
S_{L}=\pi L^{2}
$$




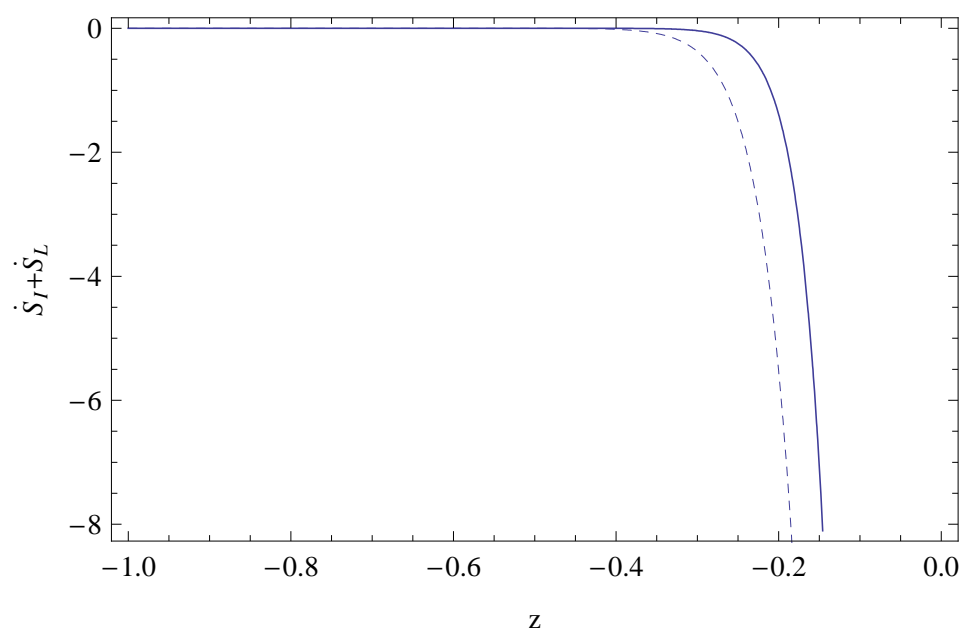

Fig.3

Fig. 3 shows the variation of $\left(\dot{S}_{I}+\dot{S}_{L}\right)$ against redshift $z$ for $c=.5, \rho_{m_{0}}=1, \alpha=.7, \beta=.7, H_{0}=70$. The dash line and non-dash line represent for the generalized holographic and generalized Ricci dark energy models respectively.

The amount of the energy crossing on the horizon is (same as (24)) given by [25]

$$
-d E_{L}=4 \pi L^{3} H T_{\mu \nu} k^{\mu} k^{\nu} d t=4 \pi L^{3} H(\rho+p) d t=-H \dot{H} L^{3} d t
$$

and from (52) and (53) we have

$$
T_{L} d S_{L}=\dot{L} d t
$$

From (54) and (55), we obtain the relation

$$
-d E_{L}=T_{L} d S_{L}-\left(H \dot{H} L^{3}+\dot{L}\right) d t
$$

The second term on the r.h.s. is time dependent, so this term cannot become zero during certain stage of the evolution of the universe. Thus we may conclude that

$$
-d E_{L} \neq T_{L} d S_{L}
$$

So on the horizon, first law of thermodynamics can not be satisfied in Einstein's gravity.

Now on the horizon, the rate of change of total entropy is (similar to (38)) obtained as

$$
\dot{S}_{I}+\dot{S}_{L}=2 \pi L\left[\dot{H} L^{2}(H L-\dot{L})+\dot{L}\right]
$$

The GSL will be satisfied if

$$
\dot{H} L^{2}(H L-\dot{L})+\dot{L} \geq 0
$$

For quintessence model $(\dot{H}<0)$ or phantom model $\dot{H}>0$ or phantom crossing model, we can not draw any definite conclusion for validity of GSL on the horizon. The validity is completely depends on the value of $L$ in 
the dark energy model. In generalized holographic dark energy model, the rate of change of total entropy on the horizon is calculated as (from (10), (11) and (58))

$$
\begin{gathered}
\dot{S}_{I}+\dot{S}_{L}=\pi H\left[\dot{H} L^{2}\left(2 L^{2}+(1+z) \frac{d L^{2}}{d z}\right)-(1+z) \frac{d L^{2}}{d z}\right] \\
=\pi\left[\frac{8 \pi \rho_{m_{0}}(1+z)^{3}}{3\left(1-c^{2}+4 \alpha c^{2}\right)}+H_{0}^{2}(1+z)^{\frac{c^{2}(13 \alpha-1)+1}{3 \alpha c^{2}}}\right]^{\frac{1}{2}} \times\left[-(1+z) \frac{d L^{2}}{d z}+\right. \\
\left.+\left\{-\frac{8 \pi \rho_{m_{0}}(1+z)^{3}}{2\left(1-c^{2}+4 \alpha c^{2}\right)}+\frac{c^{2}(1-13 \alpha)-1}{6 \alpha c^{2}} H_{0}^{2}(1+z)^{\frac{c^{2}(13 \alpha-1)+1}{3 \alpha c^{2}}}\right\} L^{2}\left(2 L^{2}+(1+z) \frac{d L^{2}}{d z}\right)\right]
\end{gathered}
$$

where $L^{2}$ has the expression given in equation (51). This is very complicated expression in $z$, so we need graphical investigation. The rate of change of total entropy on the horizon of radius $L$ have drawn in fig. 3 . So Fig. 3 shows the variation of $\left(\dot{S}_{I}+\dot{S}_{L}\right)$ against redshift $z$ for $c=.5, \rho_{m_{0}}=1, \alpha=.7, \beta=.7, H_{0}=70$. The dash line and non-dash line represent for the generalized holographic and generalized Ricci dark energy models respectively. From the figure, we see that the GSL can not be satisfied on the horizon for generalized holographic and Ricci dark energy models.

\section{DISCUSSIONS}

We have considered the flat FRW model of the universe which is filled with the combination of dark matter and dark energy. Here we have considered two types of dark energy models: (i) Generalized holographic and (ii) generalized Ricci dark energies. If $\beta=1-\alpha$, the generalized Ricci dark energy model is converted to generalized holographic dark energy model. When $\alpha=0$ or $\beta=1$, we recover the energy densities of original holographic dark energy. Also when $\alpha=1$ or $\beta=0$, we recover the energy density of original Ricci dark energy. In these two models, the solutions have been found in terms of the redshift $z$. The general descriptions of first law and generalized second law (GSL) of thermodynamics have studied on the apparent horizon, particle horizon and event horizon of the universe. Here we have tried to apply the usual definition of the temperature and entropy as that of the apparent horizon to the particle horizon and the cosmological event horizon and examine the validity of first and the second laws of thermodynamics. We have shown that the first law and GSL are always valid on apparent horizon and first law can not be satisfied on the particle and event horizons in Einstein's gravity. These results are always true for any types of dark energy models i.e., these results do not depend on the dark energy models in Einstein's gravity. But the GSL completely depends on the choices of dark energy models in Einstein's gravity. Here we have discussed the validity of GSL in Generalized holographic and generalized Ricci dark energy models. From figures 1 and 2, we have seen that the GSL may be satisfied on the particle horizon and can not be satisfied on the event horizon for both generalized holographic and Ricci dark energy models. Also we have considered the Generalized holographic dark energy and generalized Ricci dark energy as the original holographic dark energy, so in this situation we have calculated the expression of the radius of the horizon $L$ in terms of redshift $z$. On this horizon, we have shown that the first law can not be satisfied. From figure 3, we have seen that the GSL can not be satisfied on the horizon of radius $L$ for both dark energy models.

\section{Acknowledgement:}

The authors are thankful to IUCAA, Pune, India for warm hospitality where part of the work was carried out.

\section{References:}

[1] S. J. Perlmutter et al, Bull. Am. Astron. Soc. 291351 (1997); S. Perlmutter et al, Nature 39151 (1998); S. J. Perlmutter et al, Astrophys. J. 517565 (1999).

[2] A. G. Riess et al, Astron. J. 1161009 (1998); P. Garnavich et al, Astrophys. J. 493 L53 (1998); B. P. 
Schmidt et al, Astrophys. J. 50746 (1998); N. A. Bachall, J. P. Ostriker, S. Perlmutter and P. J. Steinhardt, Science 2841481 (1999).

[3] V. Sahni and A. A. Starobinsky, Int. J. Mod. Phys. A 9373 (2000); P. J. E. Peebles and B. Ratra, Rev. Mod. Phys. 75559 (2003).

[4] T. Padmanabhan, Phys. Rept. 380235 (2003); E. J. Copeland, M. Sami, S. Tsujikawa, Int. J. Mod. Phys. $D 151753$ (2006); J. A. Frieman, M. S. Turner and D. Huterer, arXiv:0803.0982 astro-ph].

[5] B. Ratra and P. J. E. Peebles, Phys. Rev. D 373406 (1988).

[6] T. Chiba, T. Okabe and M. Yamaguchi, Phys. Rev. D 62023511 (2000).

[7] A. Sen, JHEP 0204048 (2002).

[8] A. Kamenshchik, U. Moschella and V. Pasquier, Phys. Lett. B 511265 (2001); V. Gorini, A. Kamenshchik, U. Moschella and V. Pasquier, gr-qc/0403062; V. Gorini, A. Kamenshchik and U. Moschella, Phys. Rev. D 67 063509 (2003); U. Alam, V. Sahni, T. D. Saini and A. A. Starobinsky, Mon. Not. R. Astron. Soc. 344, 1057 (2003); H. B. Benaoum, hep-th/0205140; U. Debnath, A. Banerjee and S. Chakraborty, Class. Quantum Grav. 215609 (2004).

[9] R. R. Caldwell, Phys. Lett. B 54523 (2002).

[10] E. Witten, hep-ph/0002297.

[11] A. G. Cohen, D. B. Kaplan and A. E. Nelson, Phys. Rev. Lett. 824971 (1999).

[12] X. Zhang, Int. J. Mod. Phys. D 141597 (2005).

[13] W. Fischler and L. Susskind, hep-th/9806039.

[14] M. R. Setare, Phys. Lett. B 648329 (2007); Y. Gong, Phys. Rev. D 70064029 (2004).

[15] M. Li, Phys. Lett. B 6031 (2004).

[16] C. Gao, F. Wu, X. Chen and Y. -G. Shen, Phys. Rev. D 79043511 (2009).

[17] C. -J. Feng, Phys. Lett. B 670231 (2008); Phys. Lett. B 67294 (2009); arXiv: 0806:0673 [hep-th]; C. -J. Feng and X. -Z. Li, Phys. Lett. B 680355 (2009); L. Xu, W. Li and J. Lu, Mod. Phys. Lett. A 241355 (2009); M. Suwa and T. Nihei, Phys. Rev. D 81023519 (2010); K. W. Kim, H. W. Lee and Y. S. Myung, arXiv: 0812:4098 [gr-qc].

[18] L. Xu, J. Lu and W. Li., Eur. Phys. J. C 6489 (2009).

[19] H. M. Sadjadi, JCAP 02026 (2007); H. M. Sadjadi and M. Honardoost, Phys. Lett. B 647231 (2007).

[20] K. Karami and A. Abdolmaleki, arXiv: 0909.2427 [gr-qc]; K. Karami and S. Ghaffari, Phys. Lett. B 685 115 (2010).

[21] Y. Zhang, Z. Yi, T. -J. Zhang and W. Liu, Phys. Rev. D 77023502 (2008).

[22] B. Wang, Y. G. Gong and E. Abdalla, Phys. Rev. D 74083520 (2006); G. Izquierdo and D. Pavon, Phys. Lett. B 633420 (2006).

[23] R. Bousso, Phys. Rev. D 71064024 (2005).

[24] S. Bhattacharya and U. Debnath, arXiv: 1006.2600[gr-qc]; arXiv: 1006.2609 [gr-qc].

[25] M. R. Setare and S Shafei, JCAP 09011 (2006); M. R. Setare, JCAP 01023 (2007). 